\title{
An Agent-Based Approach for the Design of the Future European Air Traffic Management System
}

\author{
Martin Molina, Jorge Martin, and Sergio Carrasco \\ Department of Artificial Intelligence \\ Technical University of Madrid \\ Campus de Montegancedo S/N 28660 Madrid, Spain \\ martin.molina@upm.es, \{scarrasco,jmartin\}@fi.upm.es
}

\begin{abstract}
This paper describes an agent-based approach for the simulation of air traffic management (ATM) in Europe that was designed to help analyze proposals for future ATM systems. This approach is able to represent new collaborative decision processes for flow traffic management, it uses an intermediate level of abstraction (useful for simulations at larger scales), and was designed to be a practical tool (open and reusable) for the development of different ATM studies. It was successfully applied in three studies related to the design of future ATM systems in Europe.
\end{abstract}

Keywords: Agent-based modeling, agent-based simulation, air traffic management system.

\section{Introduction}

In recent years, important initiatives have been developed to modernize air traffic management (ATM) systems. For example, the SESAR (Single European Sky ATM Research) program is an ambitious research and development initiative funded by the European Union, Eurocontrol and industry. The ultimate goal of SESAR is to develop a future ATM system for Europe, ensuring the safety and fluidity of air transport over the next thirty years, making flying more environmentally friendly and reducing the costs of air traffic management [4].

This paper summarizes the results of our research work $^{1}$ under the CASSIOPEIA project (Complex Adaptive Systems for Optimization of Performance in ATM) that that we developed in the context of the SESAR program. In our approach, we developed a solution to simulate new collaboration strategies of ATM stakeholders in large geographic areas at an intermediate level of abstraction that is between the microscopic and macroscopic level. Our approach was developed as a practical tool that is open and reusable for different ATM problems, and it was applied successfully in three different ATM studies.

\footnotetext{
${ }^{1}$ See more details about our agent-based approach in [3].

Y. Demazeau et al. (Eds.): PAAMS 2014, LNAI 8473, pp. 359-362, 2014.

(C) Springer International Publishing Switzerland 2014
} 


\section{Main Purpose}

The goal of the CASSIOPEIA project project was to propose a modeling approach, using techniques of complex systems and paradigms of computer science, that could provide policy-makers with the means to understand and explore initiatives that affect complex ATM networks, allowing them to test potential concepts, regulations and mechanisms to manage delay propagation, capacity limits, network congestion, and other ATM phenomena. This project was envisioned as a solution to facilitate an understanding of the cause-effect relation between policy decisions in different sectors of aviation and air traffic performance for different scopes and scales of application of regulations.

In general, agent-based approaches have been successfully applied to model ATM systems [1] [2] [5]. However, the design of future ATM systems, as it is defined by the goals of the SESAR programme in Europe, presents new challenges in agentbased modeling and simulation such as: (1) modeling new decision levels in ATM systems (such as strategic decisions related to flow and capacity management with longer temporal horizons), (2) designing new representation methods to simulate at a larger scale (e.g., multinational geographic areas in Europe) taking into account limitations concerning existing data, and (3) creating new practical tools (more easily available to the research community) to support the development of new ATM studies. In the following sections, we summarize our agent-based approach in CASSIOPEIA that we designed that addresses these challenges.

\section{The Agent-Based Approach and Applications}

The agent-based model in the CASSIOPEIA project includes agents corresponding to different ATM stakeholders. For example, there are agents such as network managers, airlines (with agent subclasses: network airline, cargo airline, low-cost airline, etc.), airports (with information such as geographic location, category, etc.), and aircraft (with information such as model, capacity, $\mathrm{CO}$ emissions, weight, etc.). The model also includes objects related to the environment and general decision-making processes such as flight plans, time slots, and geographical sectors. The agent models follow a BDI approach, with beliefs, goals and plans.

This model includes algorithms to simulate collaborative decision-making processes corresponding to future ATM systems. For example, we implemented algorithms that simulate how airlines interact to bid and sell air traffic slots to reschedule flight plans with lower costs. We follow an intermediate level abstraction with a stochastic approach to simulate certain air traffic processes. For example, we follow this approach to simulate how airlines coordinate aircrafts in the presence of delays. The stochastic approach is used to simulate the movement of aircraft between airports, abstracting details about delays [3].

This agent-based approach was used in three different studies in the CASSIOPEIA project: (1) analyze the effects of new environmental regulations (e.g., restrictions of night traffic at certain airports to reduce noise pollution), (2) analyze the effect of capacity constraints considering as a new strategy that airlines can exchange traffic slots, and (3) analyze the effect of new methods of speed adjustment for aircraft based 
on environmental conditions. For these studies, we used data on air transportation in Europe from several sources. The majority of the data was acquired from ALL-FT+, a dataset collected by the PRISME group from Eurocontrol. Each study included several simulations with different input data corresponding to various scenarios. For example, for the first study, we used data from 79,852 flights, 838 airports and 84 airlines and generated approximately 4,500 interaction messages (during one of the simulations).

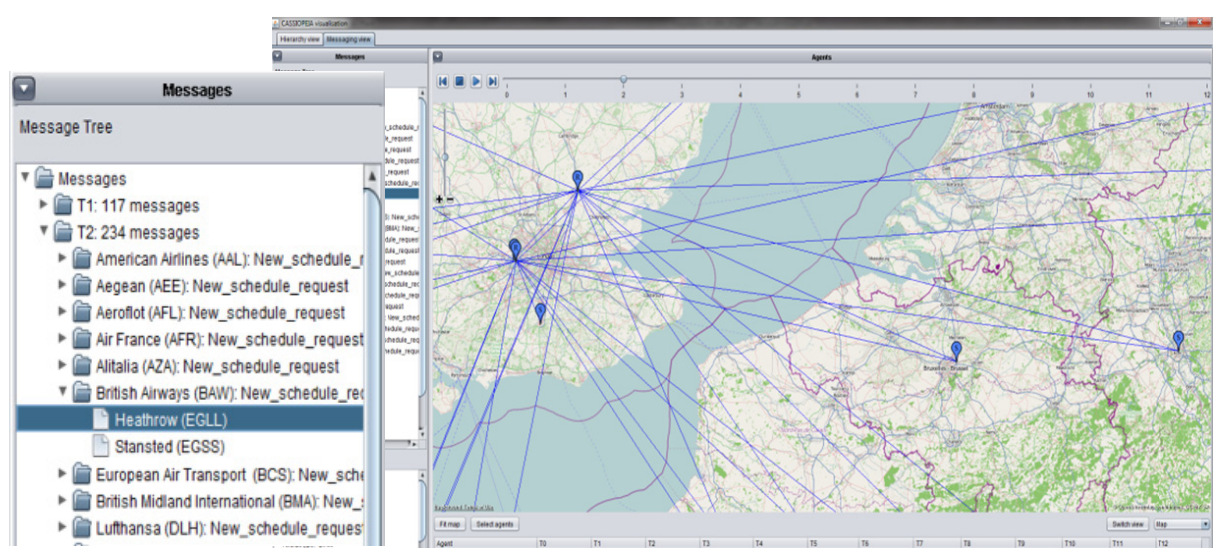

Fig. 1. Visualization tool showing agent interaction messages

The software architecture in the CASSIOPEIA project was designed to be a general tool for use in various ATM studies. Agent models are specified using the XML declarative language, together with algorithms implemented in the Java language for agent plans, with reusable libraries of classes. The software architecture was developed by integrating open software tools (Jadex, MySQL, OpenStreetMaps, etc.) to facilitate reusability at reduced costs. The architecture includes a visualization tool, developed in this project, to visualize complex agent interactions with large numbers of messages. This tool aggregates information using different dimensions (spatial, temporal, types of messages, types of agents, etc.) and presents the information using animations on geographic maps and specific types of hierarchies and tables (Figure 1). The software architecture was reused to develop the agent models for the three studies. We reused $78 \%$ of the agent specifications in the XML language, $72 \%$ of the Java code and $91 \%$ of the database design.

\section{Conclusions}

The design of future ATM systems, as it is defined by the SESAR program in Europe, presents new challenges for agent-based modeling and simulation. We designed an agent-based approach in the CASSIOPEIA project to address theses challenges with new algorithms for distributed-collaboration decision making and intermediate abstraction levels for simulations using stochastic approaches. Our software architecture 
in CASSIOPEIA was designed as a general tool and was used in three ATM studies with high percentages of reuse (more than $70 \%$ ) of the different components. In comparison to previous related studies, we simulated new ATM decision processes, and they were applied to larger areas (with hundreds of airports and longer temporal scales).

Acknowledgements. The research presented in this paper was carried out under the CASSIOPEIA project. This project received financial support from Eurocontrol under the SESAR program (Single European Sky ATM Research). The consortium members of the CASSIOPEIA project were the Innaxis Research Institute, the Technical University of Madrid (Department Artificial Intelligence and Department of Air Transport) and the University of Westminster (Department of Transport Studies). The authors would like to thank a member of our research group, Nataliia Stulova, for the implementation of the visualization tool.

\section{References}

1. Agogino, A.K., Tumer, K.: A multiagent approach to managing air traffic flow. Autonomous Agents and Multi-Agent Systems 24(1), 1-25 (2012)

2. Campbell, K., Cooper, W., Greenbaum, D., Wojcik, L.: Modeling Distributed Human Decision-Making in Traffic Flow Management Operations. In: Third USA/Europe Air Traffic Management Research and Development Seminar. The MITRE Corporation, McLean (2000)

3. Molina, M., Carrasco, S., Martin, J.: Agent-Based Modeling and Simulation for the Design of the Future European Air Traffic Management System: The Experience of CASSIOPEIA. In: Corchado, J.M., et al. (eds.) PAAMS 2014 Workshops. CCIS, vol. 430, pp. 22-33. Springer, Heidelberg (2014)

4. Sesar: Single European Sky ATM Research, http: / /www . sesarju . eu

5. Wolfe, S., Jarvis, P., Enomoto, F., Sierhuis, M., Putten, B., Sheth, K.: A Multi-Agent Simulation of Collaborative Air Traffic Flow Management. In: Bazzan, A., Klugl, F. (eds.) Multi-agent Systems for Traffic and Transportation. IGI Global Publishing (2009) 